\title{
Modelo de indução de diarréia osmótica em bezerros holandeses
}

\author{
Model for osmotic diarrhea in holstein calves
}

\section{Marta Lizandra do Rego Leal ${ }^{\mathrm{I}}$ Fernanda Cavallini Cyrillo" ${ }^{\mathrm{II}}$ Clara Satisuki Mori ${ }^{\mathrm{II}}$ Lilian Emy dos Santos Michima ${ }^{\mathrm{II}}$ Marcílio Nichi ${ }^{\mathrm{II}}$ Enrico Lippi Ortolani ${ }^{\mathrm{II}}$ Fernando José BenesiI}

\section{RESUMO}

Com o objetivo de avaliar um protocolo de diarréia osmótica induzida, foram utilizados 18 bezerros hígidos, com idade entre oito e 30 dias de vida, e peso variando de 37 a $50 \mathrm{~kg}$. A diarréia e a desidratação foram induzidas por meio da administração de leite integral $\left(16,5 \mathrm{~mL} \mathrm{~kg}^{-1}\right)$, sacarose $\left(4 \mathrm{~g} \mathrm{~kg}^{-1}\right)$, espirolactona e hidroclorotiazida $\left(2 \mathrm{mg} \mathrm{kg}{ }^{-1}\right), a$ cada oito horas, durante dois dias. $O$ exame físico $e$ as coletas de sangue para determinações de componentes do hemograma, hemogasometria e de constituintes bioquímicos foram realizados em T0 (0h), T1 (24hi) e T2 (48hi). O protocolo de indução da diarréia obteve $100 \%$ de eficiência, produzindo diarréia aquosa e desidratação intensa (13\% do peso corpóreo) acompanhadas de azotemia pré-renal, aumento nos valores do hematócrito, hemoglobina e proteína total, hipercalemia, hiperlactemia, hiperfosfatemia, acidose metabólica e diminuição do défict de volume plasmático e da pressão venosa central.

Palavras-chave: bezerro, indução, diarréia osmótica.

\section{ABSTRACT}

Eighteen health Holstein calves between 18 and 30 days old and 37 weighting $50 \mathrm{~kg}$ (body weight - BW) were used to develop a model for inducing osmotic diarrhea in order to cause a status of dehydration. Thus, sucrose $(4 \mathrm{~g} \mathrm{~kg}$ $\left.B W^{-1}\right)$ and spirolactone and hydrochlorothiazide (2mg $\left.\mathrm{g} \mathrm{BW}^{-1}\right)$ were added to the whole milk $\left(16.5 \mathrm{~mL} \mathrm{~kg}^{-1}\right)$ each $8 \mathrm{~h}$ for two days. Physical examination and blood samples for hemogram, acid:base status and biochemical analysis were obtained at 0 (T0), $24 \mathrm{~h}$ (T1) and 48h (T2). Watery diarrhea and severe dehydration (13\% of the BW) were seen in all calves, followed by pre-renal azotemia, high values of hematocrit, hemoglobin and total protein levels, hiperkalemia, hyperlactemia, and hyperphosphatemia, metabolic acidosis and decreased plasma volume and central venous pressure.

Key words: calves, induction, osmotic diarrhea.

\section{INTRODUÇÃO}

A pecuária nacional, particularmente a bovinocultura, apresenta vários pontos de estrangulamento, sendo um deles a mortalidade de bezerros no primeiro mês de vida, a qual contribui de modo significativo para aumentar os custos de produção. Apesar de, nos últimos anos, a Medicina Veterinária ter apresentado considerável evolução tecnológica em várias áreas de atuação, não se observam reflexos consistentes na redução da taxa de mortalidade de bezerros neonatos. Entre as principais causas de óbito, nessa categoria animal, destaca-se a diarréia, como responsável por grandes prejuízos econômicos na atividade pecuária mundial (SIMONS \& BYWATER, 1991; BENEDETI et al., 2000; NAYLOR et al., 2006; LORENZ \& VOGT, 2007). Sabe-se que a morte de bezerros em decorrência da diarréia se dá principalmente pelos desequilíbrios hidroeletrólitico e ácido-base (WALKER et al., 1998; NAYLOR, et al., 2006; LORENZ \& VOGT, 2007), sendo a correção dessas alterações a chave para minimizar a perda de bezerros por esta síndrome. No entanto, é notória a escassez de pesquisas nacionais dedicadas a esse assunto. Há

'Departamento de Grandes Animais, Universidade Federal de Santa Maria (UFSM). Avenida Roraima, 1000, 97105-900, Santa

Maria, RS, Brasil. E-mail: mlizandra@smail.ufsm.br. Autor para correspondência.

"Departamento de Clínica Médica, Faculdade de Medicina Veterinária e Zootecnia, Universidade de São Paulo (FMVZ/USP), São Paulo, SP, Brasil.

IIIDepartamento de Reprodução, FMVZ, USP, São Paulo, SP, Brasil. 
apenas um trabalho relacionado ao tratamento da diarréia em bezerros neonatos com uso da fluidoterapia oral e parenteral (FERREIRA, 2001). Assim é de extrema importância a existência de novas pesquisas acerca de métodos de indução experimental da diarréia em bezerros, bem como o estudo de novas formas de tratamento a fim de minimizar as perdas geradas por essa enfermidade na pecuária nacional. Vários são os métodos utilizados para induzir diarréia em bezerros neonatos. Esses métodos podem ser divididos em dois grupos: infecciosos e não-infecciosos. O modelo infeccioso para a indução da diarréia inclui o uso de cepas de Escherichia coli enterotoxigênica (HEATH et al., 1989), Criptosporidium parvum (TZIPORI et al., 1983), coronavírus (SAIF et al., 1986), rotavírus ou combinação de agentes infecciosos (NAYLOR, 1987). Os métodos não-infecciosos que têm sido utilizados, por via oral, para causar diarréia em bezerros são administração de sacarose (CONSTABLE et al., 1996; WALKER et al., 1998; CONSTABLE et al., 2000; FERREIRA, 2001), de lactose (HUBER et al., 1961), de sorbitol (NAPPERT et al., 1993), de manitol (FIORAMONT \& BUENO, 1987), de neomicina, de ampicilina e tetraciclina (HUFFMAN et al.; 1981; MERO et al.,1985) e de toxinas purificadas da Escherichia coli (ROUSSEL et al., 1988). Apesar de cada um desses modelos, com o uso de agentes infecciosos ou não, serem capazes de causar diarréia, a maioria não preenche os critérios desejáveis para um modelo ideal de indução da diarréia. Os problemas que apresentam incluem: grande variação da resposta, alta mortalidade de bezerros (HEATH et al., 1989) e um tempo indeterminado para o início da diarréia (TZIPORI et al.,1983; SAIF et al.,1986;). Essas variações aumentam o número de bezerros necessários para comparar diferentes modalidades de tratamentos, além de aumentarem os custos da indução (CONSTABLE et al., 1996; WALKER et al., 1998). Considerando a significância econômica desta síndrome, reveste-se de grande importância a avaliação de um modelo de indução de diarréia prático e econômico, que permita comparar, de forma segura, diferentes protocolos de tratamento, bem como estudar a patofisiologia da diarréia neonatal. O presente estudo teve por objetivo avaliar a eficácia de um modelo de indução de diarréia em bezerros neonatos.

\section{MATERIAL E MÉTODOS}

Foram utilizados 18 bezerros hígidos, da raça Holandesa, com idade entre oito e 30 dias, e peso corporal de 37 a $50 \mathrm{~kg}$, provenientes de propriedades leiteiras, localizadas no município de Itapetininga, no
Estado de São Paulo. Os bezerros foram alojados individualmente em gaiolas galvanizadas instaladas em baias localizadas na Clínica de Bovinos do Departamento de Clínica Médica da Faculdade de Medicina Veterinária e Zootecnia da Universidade de São Paulo, sendo alimentados em mamadeiras com quatro litros de leite integral ${ }^{\mathrm{a}}$ (130g por litro de água) ao dia, divididos em dois repastos (às 8 e 17h), além de terem água disponível para consumo “ad libitum”. Antes de o início do experimento, os bezerros foram mantidos em suas instalações por um período mínimo de cinco dias para a adaptação à dieta e ao local de permanência. Os animais selecionados para o experimento foram considerados hígidos após exame físico (DIRKSEN et al., 1993), hemograma completo e exames eletrocardiográfico ${ }^{\mathrm{b}}$ e ecocardiográfico ${ }^{\mathrm{c}}$.

No dia que antecedeu o início do experimento, foi implantado um cateter intravenoso ${ }^{\mathrm{d}}$ nos animais por meio do qual foi mensurada a pressão venosa central e coletadas as amostras sangüíneas, para as análises programadas no estudo. Para implantação do cateter realizou-se a tricotomia e antisepsia da região cervical ventral e puncionou-se a veia jugular esquerda para passagem do cateter, intravenosamente, na direção do fluxo sangüíneo. Após a introdução do cateter no átrio direito, retirou-se a agulha, aplicando-se sobre ela o dispositivo protetor, fixando-a na pele com ponto simples, usando fio de náilon n. 1-0. Realizou-se a lavagem do cateter com $10 \mathrm{ml}$ de solução fisiológica com 500UI de heparina sódica ${ }^{e}$ a cada oito horas a fim de evitar a coagulação do sangue no interior do mesmo.

A diarréia osmótica e a desidratação foram induzidas por via oral mediante administração de leite integral $\left(16,5 \mathrm{~mL} \mathrm{~kg}{ }^{-1}\right)$ sacarose ${ }^{\mathrm{f}}\left(4 \mathrm{~g} \mathrm{~kg}^{-1}\right.$ - diluída em uma solução aquosa a 20\%) e de dois tipos de diuréticos: espirolactona ${ }^{\mathrm{g}}$ e hidroclorotiazida ${ }^{\mathrm{i}}\left(2 \mathrm{mg} \mathrm{kg}^{-1}\right)$ a cada oito horas por um período de 48 horas.

Utilizando-se os métodos semiológicos preconizados por DIRKSEN et al. (1993), foram aferidas as funções vitais: temperatura retal (TR) e freqüências cardíaca (FC) e respiratória (FR). O escore fecal para pontuação do nível de consistência das fezes e os graus de desidratação e de depressão clínica foram julgados por meio de índices, de acordo com a seguinte classificaçãode Walker et al. (1998). Em relação ao escore fecal: $0=$ normal: fezes bem formadas (firmes), $1=$ anormal: fezes tendendo a pastosas, mas ainda não caracterizando diarréia, 2=fezes pastosas: diarréia moderada; 3=fezes aquosas: diarréia intensa. Em relação ao grau de desidratação clínica: $0=$ normal: olhos brilhantes e tugor de pele normal, $1=$ leve: tugor de pele levemente reduzido, mucosa oral pastosa e colando, vasos episclerais 
injetados (perda de peso corporal entre 6 e 8\%), 2=moderado: globo ocular levemente retraído, diminuição evidente do tugor da pele, mucosa oral seca (perda de peso corporal entre 8 e 10\%), 3=intenso: globo ocular bem retraído, diminuição acentuada do tugor de pele, focinho e cavidade oral frios (mais de $10 \%$ de perda de peso corporal). Por fim, em relação ao grau de depressão clínica: $0=$ normal, $1=$ leve: bezerro mamando, mas não vigorosamente, $2=$ moderada: bezerro ainda fica em estação, mas a sugação é lenta ou desorganizada, 3=intensa: bezerro incapaz de mamar e de ficar em estação.

As amostras de sangue foram coletadas em seringas ${ }^{i}$, com capacidade para $20 \mathrm{ml}$, diretamente do cateter intravenoso, sendo divididas em três alíquotas. Uma destinada à determinação de componentes do hemograma (tubos contendo EDTA tripotássicoj), outra disposta em tubos siliconizados sem anticoagulante, para obtenção de soro destinado à realização de provas bioquímicas, e uma última alíquota, colocada em tubos siliconizados, contendo como conservante o oxalato e o fluoreto se sódio', que foi utilizada para determinar a glicose e o ácido láctico plasmáticos.

As amostras de sangue sem anticoagulante e aquelas contendo oxalato e fluoreto de sódio foram submetidas à centrifugação por 15 minutos, sendo logo após acondicionada em tubos de Eppendorf e congeladas a - $20^{\circ} \mathrm{C}$ até o momento das determinações bioquímicas.

Amostras de 2ml de sangue, para realização de provas hemogasométricas, foram coletadas diretamente do cateter, com utilização de seringas plásticas com capacidade para $3 \mathrm{ml}{ }^{\mathrm{m}}$ contendo heparina sódica como anticoagulante. A coleta e a manutenção das amostras de sangue, para realização de tal exame, seguiram as recomendações feitas por LISBÔA et al. (2001).

A determinação do hematócrito e da concentração de hemoglobina foram realizados segundo metodologia descrita por BIRGEL (1982). As variáveis hemogasométricas foram quantificadas em analisador automático de $\mathrm{pH}$ e gases sangüíneos ${ }^{\mathrm{n}}$. Após a introdução da alíquota de sangue no hemogasômetro, foram corrigidos os valores da hemoglobina e da temperatura do animal avaliado, pois os valores padrão destes assumidos pelo aparelho são referentes à espécie humana.

Com exceção do sódio, do cloreto, do potássio e do lactato plasmáticos, todas as demais variáveis bioquímicas (séricas e plasmáticas) foram quantificadas em analisador bioquímico automático ${ }^{\circ}$. As seguintes variáveis foram mensuradas: proteínas totais séricas, uréia sérica, creatinina sérica, cálcio sérico, fósforo inorgânico sérico e glicose plasmática.
O sódio, os cloretos e o potássio séricos foram mensurados em analisador de íons e de gases

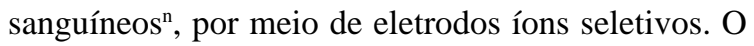
ácido láctico plasmático foi quantificado segundo metodologia empregada por PRYCE (1969).

O cálculo do déficit de volume plasmático (\%) foi efetuado segundo KANEKO et al. (1997), que relaciona por meio da expressão matemática o hematócrito do momento zero $\left(\mathrm{Ht}^{1}\right)$ com os obtidos nos tempos subseqüentes $\left(\mathrm{Ht}_{\mathrm{N}}\right)$, conforme apresentada a seguir:

Déficit de volume plasmático $(\%)=\frac{\mathrm{Ht}_{1}\left(1-H \mathrm{t}_{\mathrm{N}}\right)-1}{\mathrm{Ht}_{\mathrm{N}}\left(1-\mathrm{Ht}_{1}\right)} \times 100$

O anion-gap foi calculado a partir de fórmula descrita por KANETO et al. (2004): Aniongap=(concentração sérica de sódio + concentração sérica de potássio) - (concentração sérica de cloreto + concentração plasmática de bicarbonato)

A pressão venosa central (PVC) foi mensurada, no átrio direito dos animais em estação, de acordo com a metodologia descrita por LEAL et al. (2006). Todas as medidas seguiram a mesma técnica e foram efetuadas pelo mesmo avaliador. Em todos os animais foi realizado o ecocardiograma pelo modo- $\mathrm{B}^{\mathrm{c}}$ para confirmar a correta posição do cateter. E em um único animal, sorteado ao acaso, foi realizado o estudo radiológico cardíaco ${ }^{\mathrm{p}}$, em posição latero-lateral, com um cateter intravenoso radiopaco colocado para se estabelecer o ponto exato em que a extremidade do cateter atingia o átrio direito.

As análises estatísticas foram efetuadas com o auxílio de um programa estatístico computadorizado SAS System for Windows (SAS, 1985). Os dados foram testados quanto à normalidade dos resíduos e à homogeneidade das variâncias por meio do aplicativo Guided Data Analisys. Caso não obedecessem a essas premissas, eram transformados (logaritmo na base 10Log10x ; raiz quadrada-RQ X; Quadrado-X2).

Os dados obtidos para as variáveis que apresentaram distribuição normal foram submetidos à análise de variância (ANOVA) e ao teste de Duncan para a comparação entre pares de médias. Para as variáveis cuja normalidade não foi obtida mesmo após transformação (pressão venosa central e déficit de volume plasmático) ou para aquelas subjetivas (escore fecal, grau de desidratação e de depressão clínica), empregou-se, na análise dos resultados, o procedimento Npar1way de análise de variância não-paramétrica, utilizando-se para tal o teste de Kruskal Walis.

Para descrição dos resultados, foram empregadas as médias e os respectivos desvios-padrão 
dos dados originais para as variáveis que apresentaram distribuição normal e as medianas para aquelas cuja distribuição não foi gaussiana. O nível de significância utilizado para rejeitar H0 (hipótese de nulidade) foi de $5 \%$.

\section{RESULTADOS E DISCUSSÃO}

A escolha pelo uso da sacarose em associação com diuréticos se deu ao fato de ser um protocolo econômico e capaz de causar diarréia aquosa com desidratação em bezerros neonatos em um curto período de tempo (Tabela 1 e Figura 1), conforme observado em outras pesquisas (WALKER et al., 1998; CONSTABLE et al., 2000; FERREIRA, 2001). Em modelo utilizando sacarose e furosemida como indutores, observou-se intensa diarréia aquosa, porém, o protocolo só provocou desidratação moderada (8\%) durante as 48 horas de indução (CONSTABLE et al., 1996; FERREIRA, 2001). Todavia, o protocolo de indução da diarréia estabelecido por WALKER et al. (1998), utilizando sacarose, furosemida, hidroclorotiazida e espirolactona, causou diarréia osmótica e desidratação intensa (13\%) no período de 48 horas. No entanto, não houve evidência de depressão clínica e acidose metabólica, distúrbio caracterizado por diminuições do pH sangüíneo, bicarbonato e concentração de base sangüínea (Tabela 1 e Figura 1), desequilíbrio normalmente observado em animais com diarréia espontânea e fator primordial em casos de morte de bezerros com diarréia (NAYLOR et al., 2006; LORENZ \& VOGT, 2007). Para o presente estudo, foi estabelecida a

Tabela 1 - Valores médios e de medianas dos componentes do hemograma, hemogasometria, bioquímicos do sangue e parâmetros clínicos, avaliados em bezerros holandeses antes (0h - hora) 24 e 48 horas pós-indução (PI) da diarréia osmótica.

\begin{tabular}{|c|c|c|c|}
\hline \multirow{2}{*}{ Variáveis } & \multicolumn{3}{|c|}{ 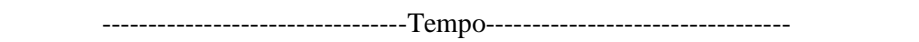 } \\
\hline & $0 \mathrm{~h}$ & 24h (PI) & $48 \mathrm{~h}(\mathrm{PI})$ \\
\hline Hematócrito (\%) & $29,33 \pm 4,45^{\mathrm{B}}$ & $34,16 \pm 4,74^{\mathrm{A}}$ & $36,14 \pm 4,36^{\mathrm{A}}$ \\
\hline Hemoglobina $\left(\mathrm{g} \mathrm{dl}^{-1}\right)$ & $9,37 \pm 1,67^{\text {B }}$ & $11,04 \pm 1,67^{\mathrm{A}}$ & $11,37 \pm 1,57^{\mathrm{A}}$ \\
\hline Proteína total $\left(\mathrm{g} \mathrm{dl}^{-1}\right)$ & $6,11 \pm 0,93^{\mathrm{C}}$ & $6,81 \pm 0,93^{\mathrm{AB}}$ & $7,11 \pm 0,88^{\mathrm{A}}$ \\
\hline Uréia $\left(\mathrm{mg} \mathrm{d}^{-1} \mathrm{l}^{-1}\right)$ & $24,84 \pm 4,75^{\mathrm{C}}$ & $35,02 \pm 5,92^{\mathrm{B}}$ & $68,56 \pm 13,44^{A}$ \\
\hline Creatinina (mg dl${ }^{-1}$ ) & $1,76 \pm 0,34^{\mathrm{C}}$ & $1,98 \pm 0,40^{\mathrm{BC}}$ & $2,38 \pm 0,39^{\mathrm{A}}$ \\
\hline Sódio $\left(\mathrm{mEq} \mathrm{l} \mathrm{l}^{-1}\right)$ & $139,56 \pm 7,80^{\text {В }}$ & $141,67 \pm 10,37^{\mathrm{AB}}$ & $142,86 \pm 13,00^{A}$ \\
\hline Cloreto (mEq l-1) & $103,84 \pm 4,87^{\text {B }}$ & $107,16 \pm 7,00^{\mathrm{AB}}$ & $109,00 \pm 9,26^{A}$ \\
\hline Potássio $\left(\mathrm{mE} \mathrm{l}^{-1}\right)$ & $5,04 \pm 0,40^{\mathrm{AB}}$ & $5,12 \pm 0,51^{\mathrm{AB}}$ & $5,59 \pm 0,80^{\mathrm{A}}$ \\
\hline Cálcio (mg dl $\left.{ }^{-1}\right)$ & $9,84 \pm 1,04^{\mathrm{A}}$ & $9,94 \pm 1,17^{\mathrm{A}}$ & $9,95 \pm 1,58^{\mathrm{A}}$ \\
\hline Fósforo $\left(\mathrm{mg} \mathrm{dl}^{-1}\right)$ & $8,73 \pm 1,59^{\mathrm{C}}$ & $9,95 \pm 1,07^{\mathrm{AB}}$ & $10,67 \pm 1,08^{A}$ \\
\hline Acido láctico plasmático $\left(\mathrm{mMol} \mathrm{l}^{-1}\right)$ & $1,36 \pm 0,58^{\mathrm{C}}$ & $1,75 \pm 0,65^{\mathrm{BC}}$ & $2,32 \pm 1,15^{\mathrm{A}}$ \\
\hline Glicose plasmática (mg dl ${ }^{-1}$ ) & $96,34 \pm 20,23^{\mathrm{AB}}$ & $90,75 \pm 20,48^{\mathrm{B}}$ & $100,45 \pm 12,64^{\mathrm{A}}$ \\
\hline $\mathrm{pH}(\mathrm{v})$ & $7,36 \pm 0,03^{\mathrm{A}}$ & $7,31 \pm 0,03^{\mathrm{B}}$ & $7,27 \pm 0,04^{\mathrm{C}}$ \\
\hline $\mathrm{cHCO}_{3}^{-}(\mathrm{v})\left(\mathrm{mMol} \mathrm{l}^{-1}\right)$ & $27,82 \pm 1,81^{\mathrm{A}}$ & $25,17 \pm 1,59^{\text {В }}$ & $21,67 \pm 2,07^{\mathrm{C}}$ \\
\hline cBase (v) $\left(\mathrm{mMol} \mathrm{l}^{-1}\right)$ & $1,54 \pm 1,45^{\mathrm{A}}$ & $-1,60 \pm 1,94^{\text {В }}$ & $-5,40 \pm 2,68^{\mathrm{C}}$ \\
\hline $\mathrm{ctCO}_{2}(\mathrm{v})\left(\mathrm{mMol} \mathrm{l}^{-1}\right)$ & $29,13 \pm 1,48^{A}$ & $26,72 \pm 1,57^{\text {в }}$ & $25,82 \pm 4,01^{\mathrm{C}}$ \\
\hline $\mathrm{pO}_{2}(\mathrm{v})\left(\mathrm{mMol} \mathrm{l}^{-1}\right)$ & $28,52 \pm 6,51^{A}$ & $26,31 \pm 5,15^{A}$ & $25,82 \pm 4,01^{\mathrm{A}}$ \\
\hline $\mathrm{pCO}_{2}(\mathrm{v})\left(\mathrm{mMol} \mathrm{l}^{-1}\right)$ & $49,62 \pm 4,52^{\mathrm{A}}$ & $50,79 \pm 3,44^{\mathrm{A}}$ & $49,18 \pm 4,92^{\mathrm{A}}$ \\
\hline Déficit de volume plasmático (\%) & $0 \pm 0^{\mathrm{A}}$ & $-18,89 \pm 7,94^{\text {B }}$ & $-24,08 \pm 12,98^{\mathrm{B}}$ \\
\hline Pressão venosa central $\left(\mathrm{cmH}_{2} \mathrm{O}\right)$ & $1,23 \pm 1,13^{\mathrm{A}}$ & $-2,17 \pm 1,25^{\mathrm{B}}$ & $-3,08 \pm 1,40^{\mathrm{C}}$ \\
\hline “anion-gap” & $12,95^{\mathrm{C}}$ & $14,53^{\mathrm{B}}$ & $17,95^{\mathrm{A}}$ \\
\hline Grau de desidratação clínica & $0 \pm 0^{\mathrm{C}}$ & $1,90 \pm 0,23^{\mathrm{B}}$ & $2,88 \pm 0,32^{\mathrm{A}}$ \\
\hline Escore fecal & $0 \pm 0^{\mathrm{B}}$ & $2,94 \pm 0,23^{\mathrm{A}}$ & $3,00 \pm 0^{\mathrm{A}}$ \\
\hline Grau de depressão clínica & $0 \pm 0^{\mathrm{C}}$ & $0,65 \pm 0,48^{\text {в }}$ & $1,19 \pm 0,38^{\mathrm{A}}$ \\
\hline Temperatura corporal $\left({ }^{\circ} \mathrm{C}\right)$ & $38,88 \pm 0,29^{A}$ & $38,81 \pm 0,30^{\mathrm{A}}$ & $38,67 \pm 0,30^{\mathrm{A}}$ \\
\hline Freqüência cardíaca (bpm) & $90,20 \pm 14,48^{\text {В }}$ & $89,20 \pm 11,91^{\text {В }}$ & $99,72 \pm 10,13^{\mathrm{A}}$ \\
\hline Freqüência respiratória (mpm) & $25,33 \pm 4,75^{\mathrm{A}}$ & $23,46 \pm 2,71^{\mathrm{B}}$ & $19,07 \pm 2,57^{\mathrm{C}}$ \\
\hline Peso $(k g)$ & $39,36 \pm 7,46^{\mathrm{A}}$ & $36,37 \pm 7,36^{\mathrm{AB}}$ & $34,29 \pm 7,31^{\mathrm{B}}$ \\
\hline
\end{tabular}

- mediana.

(v) venoso. 


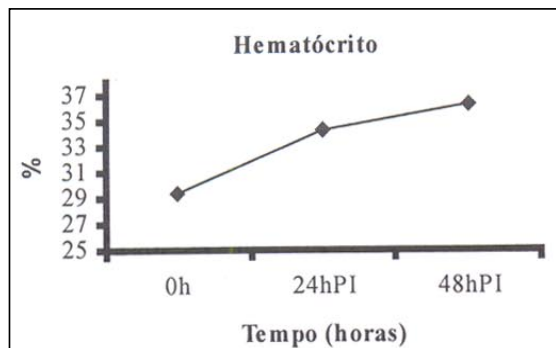

Pressão venosa central
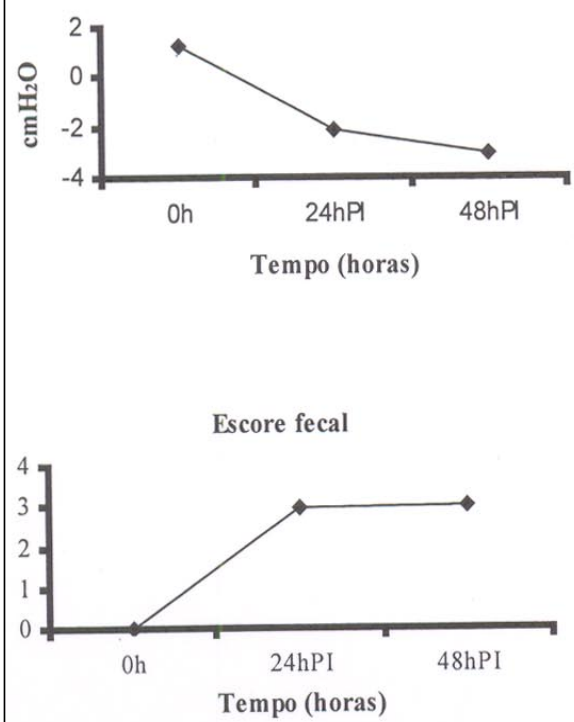

Peso corpóreo

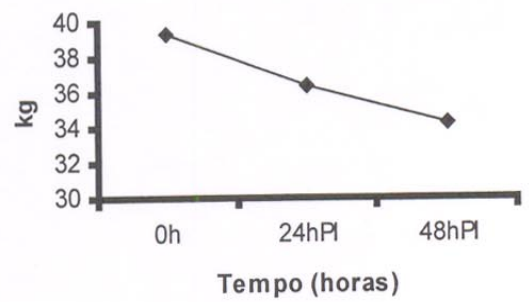

Déficit de volume plasmático

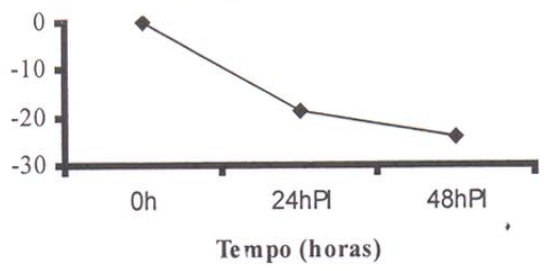

Grau de desidratação clínica

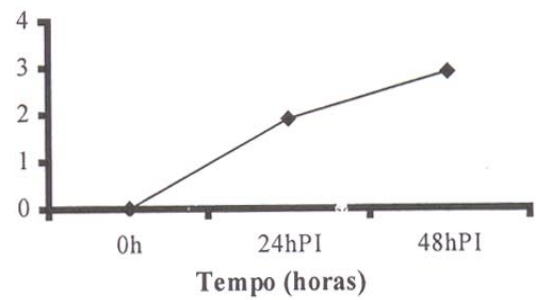

pH sanguíneo

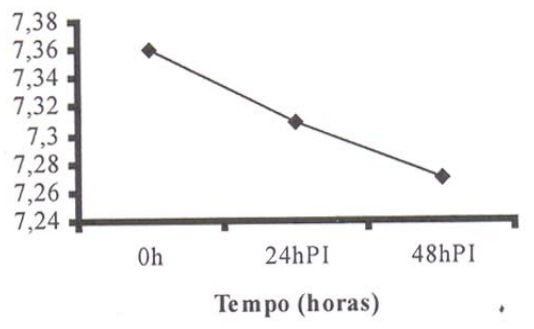

cbase

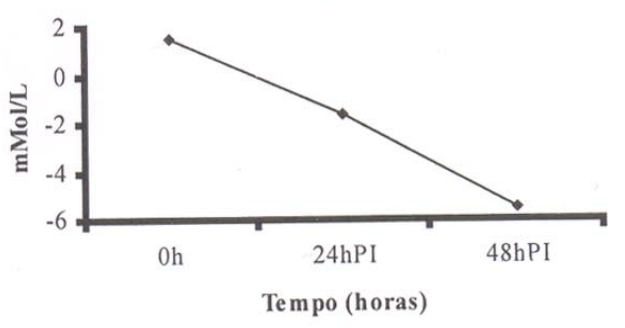

Figura 1- Valores do hematócrito, do déficit de volume plasmático, da pressão venosa central, do grau de desidratação clínica, do escore fecal, do pH sangüíneo, do peso corpóreo e da concentração de base (cBase) avaliados em bezerros holandeses antes $(0$ h), 24 e 48 horas pós-indução (PI) da diarréia osmótica.

hipótese que o uso de uma maior quantidade de sacarose aumentaria a perda de fluidos e de bicarbonato pelas fezes. A espirolactona e a hidroclorotiazida também foram utilizadas com maiores doses, contribuindo para agravar a desidratação, porém sem o risco de causar alcalose metabólica, observada quando usada furosemida(CORBETT, 1971).
O uso de sacarose e de dois tipos de diuréticos resultaram em diarréia profusa, desidratação intensa, alteração no equilíbrio hidroeletrolítico e ácidobase às 24 e 48hPI (Tabela 1 e Figura 1), com evidência de depressão moderada (Tabela 1 e Figura 1). A diarréia aquosa foi observada em todos os bezerros seis horas após a administração de sacarose e diuréticos (Tabela 
1 e Figura 1), sendo mantida durante todo o período de utilização da sacarose. Todos os bezerros mantiveram a capacidade de sugar e de sustentar-se a maior parte do tempo em estação, durante o período de indução da diarréia, apesar de apresentarem-se fracos e com moderada depressão. As extremidades do corpo apresentaram-se frias, como conseqüência da pobre perfusão periférica. O globo ocular estava evidentemente retraído e as mucosas estavam pegajosas e secas. A média de perda de peso foi de $5,1 \mathrm{~kg}$ por animal, o que correspondeu em média a aproximadamente $13 \%$ do peso corporal. Esse resultado foi maior que aquele encontrado por CONSTABLE et al., (1996) e FERREIRA (2001) e foi próximo ao obtido por WALKER et al. (1998), ao induzirem diarréia osmótica em bezerros com idade entre três e sete dias de vida.

A hipovolemia e a diminuição da taxa de filtração glomerular e do volume plasmático, em decorrência da desidratação (24 e 48hPI) (Tabela 1 e Figura 1), foram evidenciadas pelo significativo aumento de componentes do hemograma (hematócrito e hemoglobina), pela proteína total, pela uréia, pela creatinina e pelos fósforos séricos, pelo deficit de volume plasmático, pelo grau de desidratação clínica, bem como pela diminuição da pressão venosa central (Tabela 1 e Figura 1), corroborando os resultados obtidos por CONSTABLE et al. (1996), WALKER et al. (1998) e FERREIRA (2001), que utilizaram protocolos semelhantes. Quanto às funções vitais, houve um aumento dos batimentos cardíacos às $48 \mathrm{hPI}$, como provável fator compensatório da desidratação. Tammbém houve leve diminuição da freqüência respiratória e ausência de alteração na temperatura corporal (Tabela 1). O grau de desidratação e escore fecal foram máximos 48 horas após a indução da diarréia (Tabela 1 e Figura 1), enquanto que o grau de depressão clínica foi moderado (Tabela 1 e Figura 1). Desidratação em bezerros com diarréia espontânea é acompanhada por uma grande perda de volume plasmático extracelular e, em menor grau, do volume intracelular, podendo, provocar choque hipovolêmico, coma e até mesmo morte dos animais (CONSTABLE et al., 2000; FERREIRA, 2001, LORENZ \& VOGT, 2007). No presente estudo, essa alteração foi claramente observada por um aumento no déficit de volume plasmático e diminuição na pressão venosa central 48 horas após indução de diarréia (Tabela 1 e Figura 1).

A administração de sacarose, espirolactona e hidroclorotiazida mostrou-se um método eficiente e econômico na indução de diarréia em bezerros. Esse método teve um custo médio de 15 reais por animal, excluindo-se os custos com alojamento e alimentação. Outros modelos que utilizaram sacarose e um tipo de diurético (furosemida) (CONSTABLE et al., 1996; FERREIRA, 2001) ou sacarose associada a três tipos de diuréticos (furosemida, hidroclorotiazida e espirolactona) (WALKER et al., 1998) tiveram custos mais elevados, respectivamente, de 20 e 24,30 reais por bezerro.

O protocolo utilizado no presente estudo obteve $100 \%$ de resposta, pois todos os animais apresentaram diarréia e desidratação intensas em 48 horas de indução (Tabela 1 e Figura 1). A intensa desidratação não foi acompanhada por mortalidade de bezerros, porém de moderada acidemia. Todavia, em modelo de indução da diarréia utilizando a Escherichia coli, observou-se uma resposta dos animais a indução entre 53\% e 100\% dos animais, com 85\% de mortalidade em bezerros com um a quatro dias de vida (LOGAN \& PENHALE, 1972; BYWATER, 1977; DUPE et al., 1993). Em protocolo utilizando coronavírus/rotavírus, obtevese uma resposta à indução da diarréia entre 50 e 100\% dos bezerros com idade entre um a seis dias e uma mortalidade de 33\% a 86\% (LEWIS \& PHILLIPS, 1978; SAIF et al., 1986).

Estudos anteriores indicavam que bezerros com diarréia morriam quando perdiam $12 \%$ a $14 \%$ do seu peso corpóreo em decorrência da desidratação (LEWIS \& PHILLIPS,1978; GROUTIDES \& MICHEL, 1990; SIMMONS \& BYWATER, 1991). O resultado deste estudo, em concordância com o trabalho de WALKER et al.(1998), NAYLOR et al. (2006) e de NAKAGAMA et al. (2007), sugere claramente que a morte de bezerros não ocorre somente como conseqüência da desidratação mas, provavelmente, devido à associação desta a distúrbios eletrolíticos e ácido- base, especificamente acidemia intensa. Estudos indicam que a causa primária da acidose metabólica, caracterizada, no presente estudo, por diminuição do pH sanguíneo e das concentrações sangüíneas de $\mathrm{HCO}_{3}^{-}$e de cBase as 24 e 48hPI (Tabela 1 e Figura 1), é a excessiva perda de bicarbonato pelas fezes antes da desidratação intensa. Além disso, estudos indicam que esta perda é acompanhada de acidose láctica (WALKER et al., 1998; LORENZ \&VOGT, 2007, NAKAGAMA et al., 2007), como observado no presente trabalho, que revelou um aumento significativo dos teores de acido láctico plasmático às 48 horas de indução da diarréia (Tabela 1 e Figura 1), valores esses maiores que os obtidos por CONSTABLE et al. (1996) e WALKER et al. (1998) A concentração de ácido láctico plasmático pode variar com a idade do bezerro, pois animais com menos de uma semana de vida têm uma maior tendência de desenvolver acidose láctica (WALKER et al., 1998), razão pela qual optou-se por utilizar no presente estudo animais entre oito e 30 dias de vida. A acidose metabólica 
em grau intenso ocasiona sérios efeitos deletérios à função cardiovascular, inclusive redução do débito cardíaco, da pressão arterial e dos fluxos de sangue nos rins e no fígado (DIBARTOLA, 2007). Na circulação venosa, a acidose metabólica tem efeito vasoconstritor, o que tende a centralizar o volume sangüíneo e predispor a congestão pulmonar. Acidose grave também prejudica o controle do volume cerebral, provocando obnubilação e coma (DIBARTOLA, 2007. Alteração no equilíbrio acido-base também pôde ser confirmada por uma aumento significativo às 24 e $48 \mathrm{~h}$ pós-indução por meio dos valores do änion-gap”. Este simples cálculo: anion-gap $=(\mathrm{Na}+\mathrm{K})-\left(\mathrm{Cl}+\mathrm{HCO}_{3}^{-}\right)$é muito útil na categorização de desequilíbrios ácidobase com vistas aos potenciais fatores causadores (KANETO et al., 2004; DIBARTOLA, 2007). Muito comumente, as elevações de anion-gap estão associadas com o desenvolvimento de acidose metabólica em que aumentam os anions que não são rotineiramente medidos no laboratório clínico, como no caso do acúmulo de ácidos orgânicos oriundos do metabolismo (ácido láctico, ceto-ácidos) (DIBARTOLA, 2007), comumente observados nos quadros de diarréias intensas (KANETO et al., 2004). Mudanças bioquímicas observadas em bezerros com diarréia espontânea incluem azotemia pré-renal, leve hiponatremia ou hipernatremia e hiperlactemia, um grau variado de hipercalemia e hiperfosfatemia e hipoglicemia. A concentração de cloreto usualmente não muda (WALKER et al., 1998). A azotemia pré-renal (aumento nos teores séricos de uréia e creatinina) e hiperfosfatemia estão associados à desidratação e são freqüentemente observadas em bezerros com diarréia (CONSTABLE et al., 2000; LORENZ \&VOGT, 2007). O protocolo utilizado neste estudo, tendo como indutores de diarréia a sacarose e dois diuréticos, produziram desidratação intensa (13\% do peso vivo) acompanhada de azotemia pré-renal, hipercalemia, hiperlactemia, hiperfosfatemia, e moderada acidose metabólica (Tabela 1 e Figura 1), com discreto aumento nas concentrações dos íons sódio e cloretos (Tabela 1). Esses dados sugerem que este modelo experimental causou poucas diferenças no comportamento de componentes bioquímicos e ácido-base quando comparados os apresentados em animais com diarréia espontânea.

\section{CONCLUSÕES}

O protocolo de indução da diarréia em bezerros utilizando sacarose e diuréticos (espironolactona e hidroclorotiazida) mostrou-se econômico e eficiente. Com esse protocolo, houve 100\% de resposta na medida em que provocou diarréia aquosa com desidratação intensa, acompanhada de alterações nos equilíbrios hidro-eletrolítico e ácido-base, podendo ser recomendado para comparar diferentes modalidades de tratamentos da diarréia em bezerros.

\section{FONTE DE AQUISIÇÃO}

atambé ( Minas_Gerais: Brasil).

${ }^{b}$ ECG 6-Ecafix (ABMED equipamentos hospitalares: Governador Valadares, MG-Brasil),

'Sonosite 180 plus-versão1.9, (Sonoma Health Products Califórnia: EUA)

${ }^{d}$ BD- Intracath 1,7mmx30,5mm (Becton Dickinson- São Paulo: Brasil),

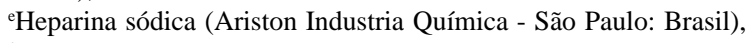
${ }^{\mathrm{f}}$ Sacarose (União-São Paulo:Brasil),

gAldactone (Pfizer-São Paulo: Brasil),

hClorana (Senofi-aventis: São Paulo-Brasil),

i, j, k, l(Becton Dickinson- São Paulo: Brasil),

${ }^{\circ}$ AVL- Omni (GMI instrumental: Copenhagem-Dinamarca), PModelo Lyasis-marca MAS (Lab. Equipment \& Supplies:Milão - Itália)

"Picker-modelo GX 1050 (São Paulo-Brasil).

\section{COMITÊ DE ÉTICA E BIOSSEGURANÇA}

Trabalho realizado de acordo com os princípios adotados pela comissão de bioética da Faculdade de Medicina Veterinária e Zootecnia da Universidade de São Paulo sob o número:1198/2007

\section{REFERÊNCIAS}

BENEDETI, E. et al. Probiótico na dieta de bezerros lactentes. Efeitos macro e microscópicos. Veterinária Notícias, v.6, n.2, p.81-88, 2000.

BIRGEL, E.H. Hematologia clínica veterinária. In: BIRGEL, E.H.; BENESI, F.J. Patologia clínica veterinária. São Paulo: Sociedade Paulista de Medicina Veterinária, 1982. p.2-49.

BYWATER, R.J. Evaluation of an oral glucose-glycine electrolyte formulation an amoxillin for treatment of diarrhea in calves. American Journal Veterinary Research, v.38, p.1983-1987, 1977.

CONSTABLE, P.D. et al. Use of hypertonic saline dextran solution to resuscitate hypovolemic calves with diarrhea. American Journal Veterinary Research, v.57, n.1, p.97104, 1996.

CONSTABLE, P.D. et al. Comparison of two oral electrolyte solutions for the treatment of dehydrated calves with experimentally induced diarrhea. Veterinary Journal, v.162, n.2, p.129-140, 2000.

CORBETT, C.E. Farmacodinâmica. 3.ed. São Paulo: Artes, 1971. 958p.

DIBARTOLA, S. Anormalidades de fluidos, eletrólitos e equilíbrio ácido-básico na clínica de pequenos animais. 3.ed. São Paulo: Roca, 2007. 664p.

Ciência Rural, v.38, n.6, set, 2008. 
DIRKSEN, G. et al. Exame clínico dos bovinos. 3.ed. Rio de Janeiro: Guanabara Koogan, 1993. 419p.

DUPE, R.J. et al. A hypertonic infusion in the treatment of experimental shock in calves and clinical shock in dogs and cats. Veterinary Record, v.133, n.24, p.585-590, 1993.

FERREIRA, F. Fluidoterapia endovenosa e oral em bezerros com diarréia osmótica induzida. 2001. $72 \mathrm{f}$. Dissertação (Mestrado em Ciência Animal) - Universidade Federal de Minas Gerais, Minas Gerais.

FIORAMONTI, J.; BUENO, L. Effects of loperamide hydrochloride on experimental diarrhea and gastrointestinal myoelectrical activity in calves. American Journal Veterinary Research, v.48, n.3, p.415-419, 1987.

GROUTIDES, C.P.; MICHELL, A.R. Changes in plasma compositions in calves surviving or dying from diarrhoea. British Veterinary Journal, v.146, n.3, p.205-210, 1990.

HEATH, S.E. et al. The effects of feeding milk to diarrheic calves supplemented with oral electrolytes. Canadian Journal Veterinary Research, v.53, n.4, p.477-485, 1989.

HUBER, T.L. et al. Utilization of carbohydrates introduced directly into the omaso-abomasal area of the stomach of cattle of various ages. Journal Dairy Science, v.44, n.2, p.321330, 1961.

HUFFMAN, E.M. et al. Serum chloramphenicol concentrations in preruminant calves: A comparison of two formulations dosed orally. Journal Pharmacology Therapy, v.4, n.2, p.225231, 1981.

KANETO, F. et al. Glicemia e anion-gap e bezerras sadias da raça holandesa no primeiro mês de vida. Ars Veterinária, v.20, n.3, p.291-298, 2004.

KANEKO, J.J. et al. Clinical biochemistry of domestic Animal. 5.ed. San Diego: Academic, 1997. 932p.

LEAL, M.L.R. et al. Pressão venosa central em bezerros hígidos. Arquivos Brasileiro de Medicina Veterinária e Zootecnia, v.50, n.4, p.525-529, 2006

LEWIS, L.D.; PHILLIPS, R.W. Pathophysiologic changes due to coronavirus-induced diarrhea in the calf. Journal of the American Veterinary Medical Association, v.173, n.5, p.636-642, 1978.

LISBÔA, J.A.N. et al. Amostras de sangue venoso bovino destinadas ao exame hemogasométrico, quando mantidas sob conservação em água gelada. Ciência Rural, v.31, n.2, p.271276, 2001.

LOGAN, E.F.; PENHALE, W.J. Studies on immunity of the calf to colibacillosis. The experimental reproduction of enteric colibacillosis. Veterinary Record, v.91, n.18, p.419-423, 1972.
LORENZ, I.; VOGT, S. Investigations on the association of Dlactate blood concentrations with the outcome of therapy of acidosis, and with posture and demeanor in yang calves with diarrhea. Journal Veterinary Diagnosis and Investigation, v,19, n.4, p.392-395, 2007

MERO, K.N. et al. Malabsortion due to selected oral antibiotics. Veterinary Clinics of North America: Food animal Practice, v.1, p.581-588, 1985.

NAKAGAMA, M. et al. Relationship between depression score and acid-base status in Japanese black calves with diarrhea. Journal Veterinary Medicine Science, v.69, n.5, p.549552, 2007.

NAPPERT, G. et al. Determination of lactose and xylose malabsortion in preruminant diarrheic calves. Canadian Journal of Veterinay Research, v.57, n.3, p.152-158, 1993.

NAYLOR, J.M. et al. Advances in oral and intravenous fluid therapy of calves with gastrointestinal disease. In: WORD BUIATRICS CONGRESS, 24., 2006, Nice, Paris. Anais.... Nice: Societé Française de Buiatrie, 2006. p.139-150.

NAYLOR, J.M. Severity and nature of acidosis in diarrheic calves over and under one week of age. Canadian Veterinary Journal, v.18, n.4, p.168-173, 1987.

PRYCE, J.D. A modification of the Barker-summerson method for the determination of lactic acid infused in to the rumen in the sheep. Bulletin Veterinary Institute Pulway, v.20, p.3-4, 1969.

ROUSSEL, A. et al. Effect of flunixin meglumine on Escherichia coli heat-stable enterotoxin -induced diarrhea in calves. American Journal Veterinary Research, v.49, n.8, p.14311433, 1988.

SAIF, L. J. et al. Experimentally induced coronavirus infections in calves: viral replication in the respiratory and intestinal tracts. American Journal Veterinary Research, v.47, n.7, p.1426-1432, 1986.

STATISTICAL ANALISYS SYSTEM. SAS use's guide: Statistics. Cary, 1985. 956p.

SIMMONS, R.D.; BYWATER, R.J. Oral rehydration in the management of neonatal diarrhea in livestock. Compendium on Continuing Education for the Practicing Veterinarian, v.13, n.2, p.345-350, 1991.

TZIPORI, S. et al. Experimental cryptosporidiosis in calves. Veterinary Record, v.112, n.5, p.116-120, 1983,

WALKER, P. et al. A reliable, practical, and economical protocol for inducing diarrhea and severe dehydration in the neonatal calf. Canadian Journal of Veterinary Research, v.62, n.3, p.205-213, 1998. 